\title{
DNA-functionalized gold nanoparticle assemblies for Surface Enhanced Raman Scattering
}

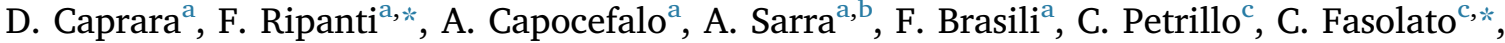 \\ P. Postorino ${ }^{\text {a, } *}$
}

a Dipartimento di Fisica, Università Sapienza, P.le Aldo Moro 5, Roma, Italy

b Dipartimento di Scienze, Università di Roma Tre, Via Ostiense, Roma, Italy

${ }^{\mathrm{c}}$ Dipartimento di Fisica e Geologia, Università degli Studi di Perugia, Via A. Pascoli, Perugia, Italy

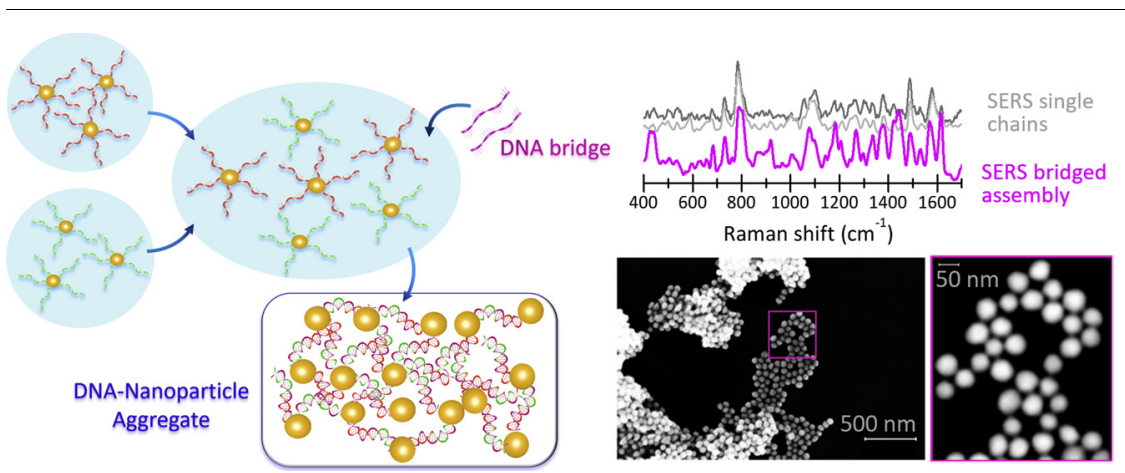

\section{A R T I C L E I N F O}

\section{Keywords:}

SERS sensor

DNA hybridization

Nanoparticle

Self-assembly

\begin{abstract}
A B S T R A C T
The programmable assembly of DNA strands is a promising tool for building tailored bottom-up nanostructures. Here, we present a plasmonic nanosystem obtained by the base-pairing mediated aggregation of gold nanoparticles (NPs) which are separately functionalized with two different single-stranded DNA chains. Their controlled assembly is mediated by a complementary DNA "bridge" sequence. We monitor the formation of DNA assembled NP aggregates in solution, and we study their Surface Enhanced Raman Scattering (SERS) response by comparison with the single NP constituents. We interpret the revealed SERS signatures in terms of the molecular and NP organization at the nanoscale, demonstrating that the action of the DNA bridge molecule yields regular NP aggregates with controlled interparticle distance and reproducible SERS response. In perspective, this demonstrates the potential of the present system as a stable, biocompatible, and recyclable SERS sensor.
\end{abstract}

\section{Introduction}

In recent years, considerable efforts have brought to the development of novel strategies for realizing targeted nanostructures by bottom-up self-assembly processes [1-3]. These represent a valid, cost- effective alternative to the established top-down nanofabrication techniques, as nanolithography or ion-beam milling [4,5]. In this framework, DNA-based nanomaterials have gained increasing attention, owing to the programmability of nucleic acid assembly: indeed, the pairing of complementary bases (adenine-thymine, cytosine-guanine)

\footnotetext{
* Corresponding authors.

E-mail addresses: francesca.ripanti@uniroma1.it (F. Ripanti), claudia.fasolato@unipg.it (C. Fasolato), paolo.postorino@roma1.infn.it (P. Postorino).
} 
enables the realization of rationally-designed materials [6-10]. Among these novel nanotechnological platforms, hybrid systems composed of DNA and noble metal plasmonic nanostructures are promising candidates $[1,3,11]$. Indeed, the possibility of coupling the self-assembly capabilities and thermal reversibility of DNA with the optical properties of metal nanoparticles (NPs) enables the realization of systems of interest for sensing applications based on Surface Plasmon Resonance (SPR) $[12,13]$ or on Surface Enhanced Raman Scattering (SERS) [14-16].

A plasmonic NP, when illuminated by light in resonance conditions with the collective oscillation of the electron gas of the metal, acts as a nanoantenna, focusing intense electromagnetic fields at the NP surface, far beyond the diffraction limit [17]. This effect can be exploited for the ultrasensitive optical spectroscopy of molecules bound/located close to the NP surface [18]. SERS spectroscopy has enabled the detection of analytes down to the femtomolar concentration range, by coupling the molecular-specific vibrational fingerprinting capability with the enhanced sensitivity of the plasmonic substrate $[14,19]$.

In some cases, the DNA-based SERS sensing schemes are aimed at revealing or investigating the DNA molecules themselves: recent works have demonstrated the ability to determine the composition of DNA strands $[15,20]$ and the DNA-metal hybridization/conjugation dynamics [21]. Single base substitutions have also been detected by SERS in single-stranded DNA chains [22,23]. In other cases, DNA has been used as an active element modulating the plasmonic efficiency of the substrate. In these applications, DNA is not the molecule directly studied by SERS, but its presence or its conformational changes trigger the SERS activity via the formation of hot-spots between NPs functionalized with standard and efficient SERS markers [24-28].

In order to promote the localization of single/double strands of DNA on the metal NP surface, overcoming the electrostatic repulsion between the negative charges of DNA and NPs, salts as $\mathrm{MgSO}_{4}$ are commonly employed [20,29]. Another possibility is the ad hoc synthesis of metal NPs stabilized by a positive ligand [15]. Indeed, the immobilization of the DNA strands on the metal surface is most commonly obtained stimulating the electrostatic attraction of the DNA by positively charged/stabilized colloidal NPs. It is otherwise possible to purchase properly designed, thiolated DNA strands and induce their covalent binding to the NP surface.

The covalent conjugation can be preferred in a series of applications, particularly those benefiting from a more stable linkage between the components of the nanosystem upon variations of temperature and $\mathrm{pH}$ of the environment. A stable conjugation is to prefer also in the perspective of designing a recyclable SERS substrate, where the analytes are typically released by chemical or physical methods after being trapped and revealed within the hot-spots of the structure [30,31]. The coverage of the NP surface by covalently immobilized ligands allows the control of the composition of the NP environment also in complex media, preventing the accidental adsorption of molecules on the NP surface, within the highly sensitive region for SERS and SPR experiments. It is worth mentioning that a covalent DNA-NP conjugation at a specific molecular concentration grants the stable and predictable orientation of the DNA strands onto the metal surface [32]. This is favorable for inducing the DNA pairing and assembly. The controlled configuration is also very important in the SERS response of the system, since the SERS spectrum of DNA strands is known to be strongly affected by the molecular orientation on the metal surface $[22,33]$.

Here, we report the study of gold (Au) NPs functionalized with two single-stranded DNA sequences. The SERS signature was characterized for the two chains by comparison with their Raman spectra to identify the spectral markers of the DNA scaffold. The self-assembly of these two hybrid DNA-NP systems within aggregates was then achieved by the action of a "bridge" molecule, in halves complementary to each of the sequences attached to the NPs. The DNA bridge acts therefore as a molecular glue. The temperature dependent UV-vis spectra acquired on the NP aggregates during their formation demonstrate that the aggregation is specific and DNA mediated. Although a specific spectroscopic marker of the DNA pairing (e.g. a band ascribed to hydrogenbonding) was not detected, a change in the system spectroscopic signature arising from the bridge molecules could be identified in the aggregated state. Notably, our study is conducted with gold NPs, while the vast majority of SERS studies on DNA rely on silver substrates $[20,21,34]$, with rare exceptions [35]. Silver nanosubstrates are in general more efficient for the SERS amplification, but poorer from the point of view of the chemical stability and biocompatibility compared to the gold counterpart $[36,37]$.

The SERS study of DNA here reported is sequence-specific, reflecting the nucleotide composition of the DNA strands used, and allows monitoring the occurrence of the NP aggregation, driven at a controlled interparticle distance by the self-assembly of DNA. The comparison between SERS spectroscopy and electron microscopy measurements demonstrates the negative correlation between the SERS signal-to-noise ratio and the interparticle distance, suggesting a path for SERS signal optimization. The present systematic study can set the basis for employing this kind of system as a DNA sensing platform, for detecting specific bridge sequences, adopting an approach similar to that in ref. [24].

Furthermore, and conveniently, the most intense SERS bands of DNA are outside the fingerprint region of common analytes detected by SERS spectroscopy $[18,38,39]$. The DNA mediated assembly of the plasmonic nanoarchitecture might be induced in presence of small analytes to detect, by trapping them within the NP aggregate and reversibly releasing them by ramping up the temperature. Provided a thorough investigation into the aggregation process in presence of analytes is conducted, the use of this system as a robust and controlled sensing platform can be envisioned.

\section{Experimental}

\subsection{Materials and characterization}

Stock solutions of $60 \mathrm{~nm}$ diameter AuNPs stabilized by citrate capping were purchased from Ted Pella, inc. (Redding, CA). Synthetic monophosphate DNA sequences with DUAL HPLC purification were purchased from Integrated DNA Technologies (IDT, USA). For the experimental realization of DNA-linked NP assemblies, we used three different and purposely-programmed single-stranded DNA chains, selected according to the seminal work of J.J. Storhoff and coworkers [40]. We refer to them as chain-A, chain-B, and bridge. Chain-A and chain-B are respectively a $5^{\prime}$ - and $3^{\prime}$-alkylthiol-capped 12 -base oligonucleotides. They were used for the functionalization of the NPs. The bridge is a non-thiolated 24-base oligomer, complementary to the chain-A and chain-B sequences. It acts as a linker for the aggregate formation. The specific sequences are

chain-A: $5^{\prime} \mathrm{X}$-S-S- $\left(\mathrm{CH}_{2}\right)_{6}$-CGCATTCAGGAT $3^{\prime}$

chain-B: $3^{\prime}$ X-S-S- $\left(\mathrm{CH}_{2}\right)_{3}$-ATGCTCAACTCT 5'

bridge: $5^{\prime}$ TACGAGTTGAGAATCCTGAATGCG $3^{\prime}$

where the X-S-S- complex specifies the thiol protecting group. Indeed, due to the high reactivity of thiol groups, during the oligonucleotide synthesis, alkylthiol-capped DNA sequences are protected by a disulfide bond; a chemical reduction is necessary for ensuring the exposition of the thiol group for the NP functionalization. The preliminary Raman characterization was performed on the pristine molecular samples. Small volumes of the three different strands in aqueous solution were dropcasted on a planar gold substrate and dried at room temperature.

\subsection{Nanoparticle functionalization}

The three DNA sequences were separately diluted in deionized MilliQ water. The actual strand concentration in these solutions was determined using a NanoDrop Microvolume Spectrophotometer 
(ThermoFisher Scientific, USA). To deprotect the thiolated sequences (chain-A and chain-B) from the disulfide bonds, we dissolved $5 \mathrm{nmol}$ of each strand in a $0.1 \mathrm{M}$ Dithiothreitol (DTT) solution, allowing the reaction to continue for 2 hours. The procedure was followed by the purification through NAP-5 column (GE Healthcare UK). AuNP functionalization with the thiol-ending nucleotides was carried out according to the well-defined assay procedure reported in Ref. [41]. Briefly, we initially prepared two different DNA-NP solutions, by separately adding an excess of thiolated DNA strands to a controlled volume of $60 \mathrm{~nm}$ AuNP stock solution. The NP dispersion was previously dialyzed for $24 \mathrm{~h}$ to remove part of the citrate buffer, thus making the NP surface cleaner and available for the DNA attachment. Phosphate buffer $(10 \mathrm{mM})$ and SDS surfactant (1\%) solutions were then added to the dispersion, for improving the wettability of the NP surface. A slow $\mathrm{NaCl}$ salting aging to a final $0.7 \mathrm{M}$ concentration [42] (done in 1 day) concluded the functionalization process. The salting aging is a critical and fundamental step that allows an optimal and homogeneous coverage of the NP surface, as it minimizes the electrostatic repulsion between the negative charges on both the oligonucleotide skeleton and the NP surface. Finally, to remove the unbound DNA strands, we centrifuged the dispersions at $15^{\circ} \mathrm{C}$ and $10,000 \mathrm{rpm}$ for $30 \mathrm{~min}$, removing the supernatant and finally adding a $0.3 \mathrm{M} \mathrm{NaCl}$ solution [32]. The purification step was repeated five times. For SERS and Field Effect Scanning Electron Microscopy (FESEM) measurements on the singlechain functionalized NPs, the purified DNA-NP solution was deposited on a glass slide/silicon substrate and dried at room temperature.

\subsection{DNA-NP controlled aggregation}

The bridge sequence mediated aggregation of the DNA-NP complexes was induced in solution and monitored by UV-vis spectroscopy. We prepared two equimolar solutions of AuNPs functionalized with chain-A and chain-B respectively. They were mixed in the presence of an excess of complementary bridge molecules. The final solution was incubated for $20 \mathrm{~min}$ in a pre-heated Memmert oven at $75^{\circ} \mathrm{C}$, then slowly cooled down to room temperature overnight. To remove the fraction of unbound bridges the solution was centrifuged at $4400 \mathrm{rpm}$ for $30 \mathrm{~min}$. Subsequently, the supernatant was removed and the sample re-dispersed in a $0.3 \mathrm{M} \mathrm{NaCl}$ solution. The purification step was repeated three times. For SERS and FESEM measurements, the aggregated NP purified dispersion was deposited on the substrate and dried at room temperature.

During the optimization of the experimental protocol, the formation of bridge-mediated NP aggregates was attempted at very different conditions in terms of NP and bridge concentration (data not shown). Variations in the NP concentration mainly affect the density and size of NP aggregates produced in solution and therefore observed on the substrate. On the other hand, the threshold bridge concentration for inducing the NP aggregation was identified as $10^{3}$ bridge molecules/ NP. The results presented in the main text are obtained at the optimal experimental conditions $\left(10^{4}\right.$ bridge molecules/NP) granting the formation of large aggregates yet stable in dispersion for a few hours.

\subsection{UV-vis spectroscopy and DNA aggregation measurements}

UV-vis absorption measurements were carried out from Jasco v-570 double ray spectrophotometer equipped with a Jasco ETC-505T Peltier thermostat which allowed to perform measurements at a controlled temperature. To monitor the DNA aggregation as a function of temperature, a volume of $\sim 250 \mu \mathrm{l}$ of the mixed functionalized NPs and bridge solution was sealed in a quartz cuvette (optical path $1 \mathrm{~mm}$ ). The system was heated up to $75^{\circ} \mathrm{C}$, well above the melting temperature of the DNA strands, calculated to be $\sim 54^{\circ} \mathrm{C}$ using NUPACK Oligo-simulator [43]. This condition ensured every sequence to be unbound from the others. The UV-vis spectra were collected ramping down the temperature to $20^{\circ} \mathrm{C}$ in steps of $5{ }^{\circ} \mathrm{C}$ (the thermalization time was $20 \mathrm{~min}$ ).
The cuvette was stirred before each acquisition, to ensure the sample homogeneity and to prevent the formation of precipitates.

\subsection{Raman/SERS spectroscopy}

Raman and SERS measurements were carried out using a Horiba HR-Evolution microspectrometer in backscattering geometry, equipped with a He-Ne laser, $\lambda=632.8 \mathrm{~nm}$ and $25 \mathrm{~mW}$ output power $(\sim 10 \mathrm{~mW}$ at the sample). Raman spectra were acquired by illuminating the molecular depositions with full laser power. Using proper optical filters, SERS spectra were collected keeping the laser power below $0.25 \mathrm{~mW}$ to avoid laser heating and sample degradation, induced by the enhanced field intensity occurring in presence of the plasmonic NPs. The detector was a Peltier-cooled charge-coupled device (CCD) and the resolution was better than $3 \mathrm{~cm}^{-1}$ thanks to a 600 grooves/mm grating with $800 \mathrm{~mm}$ focal length. The spectrometer was coupled with a confocal microscope. A $100 \times$ (N.A. $=0.9$ ) Olympus objective lens was used for the experimental acquisitions. Further details on the experimental apparatus can be found in Ref. [18]. The Raman and SERS spectra are presented after polynomial baseline subtraction. The SERS data are presented after a smooth processing (Savitzky-Golay method, 11 and 20 point windows for the single-chain and bridge-mediated aggregates, respectively). A comparison between smoothed and raw data is presented in Section F of the Supplementary Material. All the Raman and SERS spectra presented in the text are averaged over repeated, independent acquisitions. Further SERS data can be found in Section G of the Supplementary Material.

\subsection{Electron microscopy}

Samples for FESEM imaging were prepared as described above. Images were recorded using a Zeiss Auriga 405 microscope at Sapienza Nanoscience \& Nanotechnology Laboratories (SNN-Lab) of the Research Center on Nanotechnology Applied to Engineering (CNIS) of Sapienza University. The acquired frames were analyzed by Gwyddion software [44], version 2.53, for characterizing the morphology of the SERS substrates in terms of AuNP size and interparticle distance within clusters. The detailed description of the analysis procedure by employing the radial autocorrelation function of the scattering intensity is reported in Section I of the Supplementary Material.

\section{Results and discussion}

\subsection{Raman study of the DNA strands}

Preliminary Raman measurements allowed to identify the spectral fingerprint of the selected DNA sequences. In Fig. 1 we show the spectra collected as described in the Experimental section. The spectra are normalized to the most intense peak, at $\sim 789 \mathrm{~cm}^{-1}$, associated with the convolution of the vibrational modes of the cytosine and of the O-PO phosphate groups. In Figure S1 of the Supplementary Material, we show the Raman spectra of single triphosphate bases as a reference. Comparing the collected spectra with those of single bases provides a precise and complete assignment of the Raman peaks, as reported in the Supplementary Material (Table T1).

The effect of the reduction step on the chains, i.e. the transformation of the -S-S- into -S-H bond in chain-A and chain-B, can be studied by Raman spectroscopy. To do so, we measured the Raman signal before and after the cleavage of the disulfide protecting groups. We focused on the region around $\sim 2550 \mathrm{~cm}^{-1}$, where we expected to observe the $-\mathrm{SH}$ stretching Raman band. As shown in Figure S2, the -SH band is present in the spectrum of the chains only after the occurrence of the cleavage. This ensures that the chemical protocol here adopted is effective. 


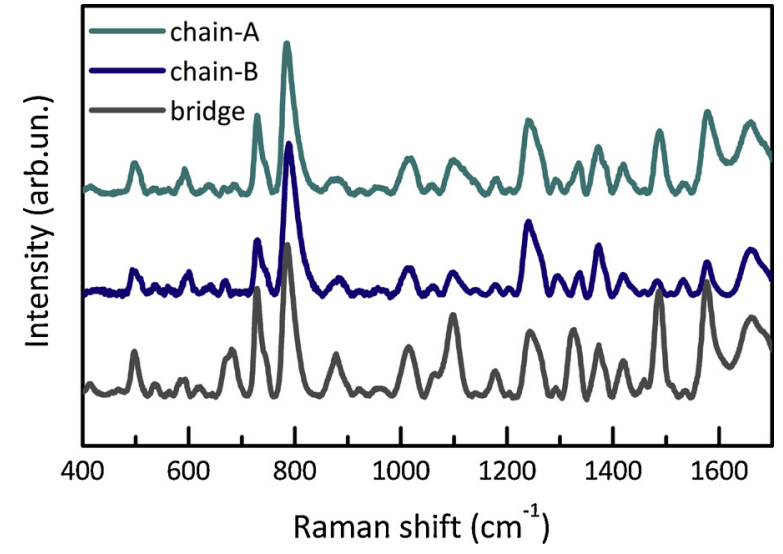

Fig. 1. Raman spectra of the single monophosphate chains: from the top, chainA (cyan), chain-B (blue), and bridge (gray). (For interpretation of the references to color in this figure legend, the reader is referred to the web version of this article.)

\subsection{DNA-AuNPs conjugation and SERS features}

The DNA anchoring on the NP surface can be monitored by UV-vis absorption measurements. In the UV-vis spectrum of the pristine plasmonic NP dispersion, a well-defined peak ascribed to the NP surface plasmon resonance can be recognized. Its position depends on the size, shape and dielectric environment around the NP. In the case of spherical AuNPs, $60 \mathrm{~nm}$ diameter, dispersed in solution, the peak is centered at $\lambda=535 \mathrm{~nm}$. When the NPs are functionalized with a ligand molecule, as DNA, the dielectric environment changes and a redshift of the plasmonic peak is observed [39]. This is verified in the functionalization with both chain- $A(\Delta \lambda \sim 6 \mathrm{~nm})$ and chain-B $(\Delta \lambda \sim 4 \mathrm{~nm})$, as shown in Fig. 2a and b respectively, which ensures a successful and stable
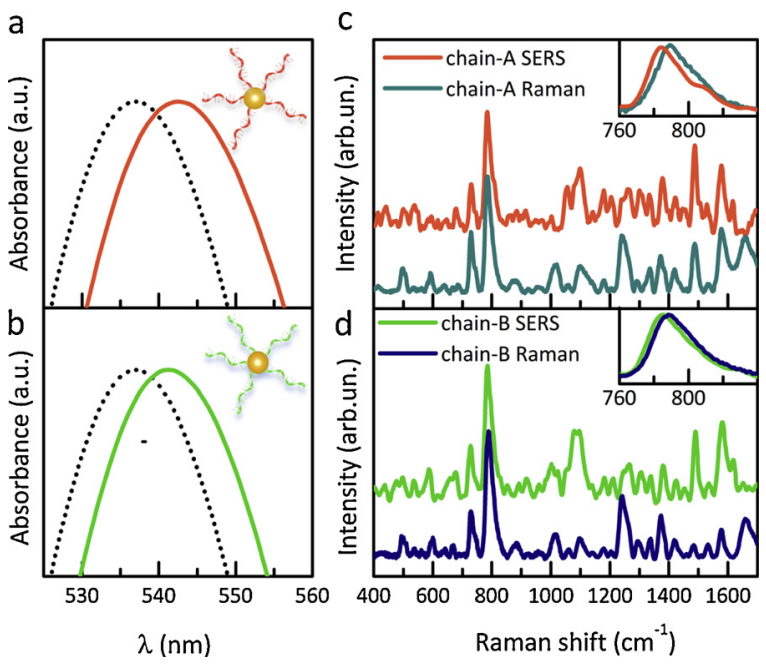

Fig. 2. Characterization of Au NPs functionalized with chain-A (top row) and chain-B (bottom row). Left column: zoom on the UV-vis absorption spectra of bare, citrate capped AuNPs (dotted black curve in panels a and b) in the region of the plasmonic absorption peak are compared to the spectrum of AuNPs functionalized with the thiolated chain-A (orange, panel a) and chain-B (green, panel b). The corresponding full spectra are reported in Figure S3 of the Supplementary Material. Spectra are normalized to the maximum of the plasmonic peak. Right column: SERS spectra of functionalized AuNPs are compared to the Raman spectra of the corresponding DNA chain-A (panel c, orange: SERS; cyan: Raman) and chain-B (panel d, green: SERS; blue: Raman). The insets highlight the redshift of the most intense SERS band (cytosine + O-P-O) as compared to the Raman spectrum. This band was used for spectral normalization. (For interpretation of the references to color in this figure legend, the reader is referred to the web version of this article.) conjugation of the AuNPs with the DNA aptamers. The complete UV-vis spectra for both the bare and the functionalized NP solutions are shown in Figure S3 of the Supplementary Material.

The SERS features of DNA-AuNPs were investigated by carrying out direct spectroscopic measurements. The good agreement between the Raman and SERS spectra of chain-A and chain-B functionalized NPs is evident from their comparison in Fig. 2 (panels $\mathrm{c}-\mathrm{d}$, respectively). Generally, a one-to-one correspondence between the SERS and Raman peaks is noticeable. As expected in SERS, the intensity ratio between the peaks is modified with respect to the Raman spectra, owing to a variable contribution of the chemical effect in the enhancement of different SERS bands [45]. A general increase in the width of the bands in the SERS spectrum is also visible. Weak redshifts of the SERS bands compared to their Raman counterparts are detected on many peaks. The most pronounced effect is observed on the most intense band of the spectrum, as shown in the insets of Fig. 2. The SERS spectral features in the region around $\sim 2550 \mathrm{~cm}^{-1}$, where the stretching vibration of the -SH group is expected, are shown in Figure S4 of the Supplementary Material. The lack of this contribution proves the absence of thiol compounds in the system, thus confirming the successful binding of the DNA molecules to the NP surface. Further, the optimized functionalization protocol minimizes the AuNP residual citrate capping, and this does not affect considerably the final SERS signal, as demonstrated by the reference spectrum in Figure S5.

The SERS response is characterized by a good reproducibility of the spectral shape, in terms of number and relative intensities of peaks. However, a marked variability $(\sim 100 \%)$ in the overall SERS intensity was retrieved during repeated acquisitions, which differ by an overall scaling factor. It is known that most of the SERS signal revealed on NPbased SERS substrates originates from the hot-spots between aggregated NPs $[45,46]$. The intensity variability is here explained by the uncontrolled self-assembly of the NPs during the drying process, which yields an irregular hot-spot size distribution.

The Raman cross-section and typical SERS enhancement factors revealed for DNA are significantly smaller than other commonly adopted SERS analytes [35]. Furthermore, the number of DNA molecules that can be loaded on a $60 \mathrm{~nm} \mathrm{NP}$ is over 10 times smaller than that of other analytes (see e.g. refs. [42] and [47]). This is an advantage for developing DNA-NP assemblies for SERS sensing, as the DNA signal would not cover that from other analytes, but it can be detrimental for obtaining structural information on the DNA scaffold. Nevertheless, despite the low intensity, the spectra in Fig. 2 allow for the recognition of the principal SERS bands, which can be assigned thanks to the comparison with the Raman counterpart (see Table T1 in the Supplementary Material). Peaks ascribed to the four bases are identified, which provides a direct fingerprint of the DNA chemical composition. The spectra are dominated by an intense peak at $\sim 784 \mathrm{~cm}^{-1}$, assigned to the vibration of the cytosine and the O-P-O phosphate group components. As highlighted in the insets of Fig. $2 \mathrm{c}$ and $\mathrm{d}$, the vibrational band observed in the Raman spectra at $\sim 789 \mathrm{~cm}^{-1}$ is redshifted in the SERS spectra for both the chains. The effect is typically observed in SERS spectra, being ascribed to the redistribution of the electronic cloud induced by molecular binding to the metal NP surface [45]. This band is used for the spectral normalization of both SERS and Raman data.

\subsection{DNA-AuNP aggregates}

Once the main components of our SERS nanosystems were characterized, we produced DNA-NP aggregates in which the bridge sequence acts as a selective linker between the functionalized NPs. The adopted experimental procedure is described in the Experimental section, and it is sketched in Fig. 3. To directly monitor the NP aggregation as a function of temperature, which leads to the formation of mesoscopic NP assemblies, UV-vis absorption measurements were performed as a function of temperature over the range $20-75^{\circ} \mathrm{C}$, starting 


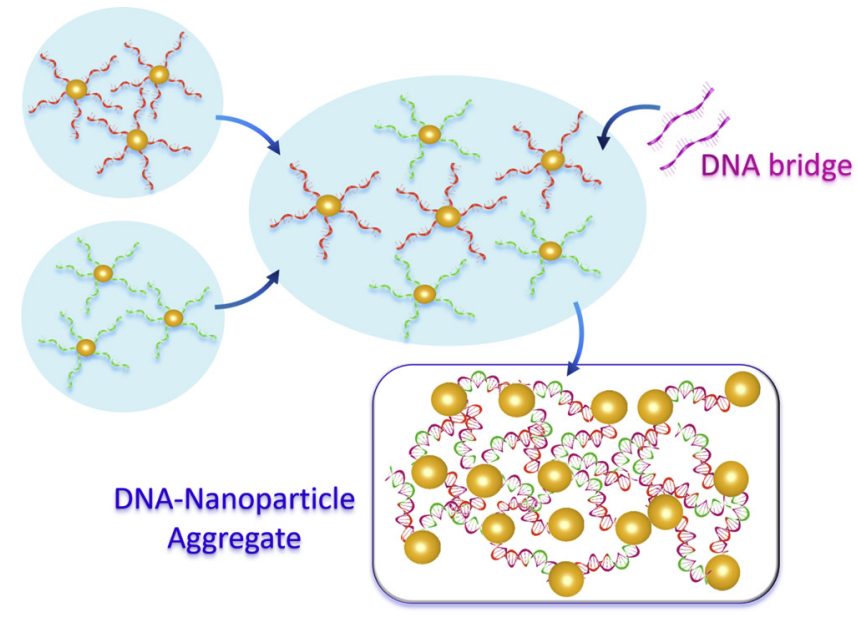

Fig. 3. Sketch of the implemented system: AuNPs, in water dispersion, are functionalized with two distinct 12-base DNA chains (-A and -B). The addition of a bridge sequence, complementary to the two chains -A and - $\mathrm{B}$, induces the system hybridization, with the formation of mesoscopic aggregates.

far beyond the DNA melting temperature and slowly cooling down the system. Thermalization times were long enough to induce the gradual pairing of the DNA sequences and, consequently, the NP assembly.

In Fig. 4 we report the UV-vis absorption spectra collected as a function of temperature during the NP aggregation by DNA pairing. At temperatures considerably higher than the system melting temperature, the UV-vis profile can be associated to a dispersion of single, DNAfunctionalized AuNPs (see Fig. 2a and b and Figure S3 in the Supplementary Material for comparison). As the temperature decreases, the complementary DNA chains pair up, forming aggregates with increasing size. The observed widening, redshift and decrease of the plasmonic absorption peak intensity, along with the increased extinction in correspondence of the red-NIR spectral region $(600-800 \mathrm{~nm})$, are expected consequences of the formation of large aggregates [39]. The temperature dependence of the observed behavior is a signature of the specificity of the DNA binding process, witnessing that the bridgemediated aggregation occurs by DNA hybridization into a double helix, which grants the NP assembly at controlled interparticle distance.

As described, the SERS characterization of the aggregates was performed measuring a microvolume of the annealed DNA-NP solution dropcasted and dried on a glass slide. The SERS spectrum of the doublestranded DNA was measured and in Fig. 5 we show the comparison between the SERS spectra of the two separated chains and that of the

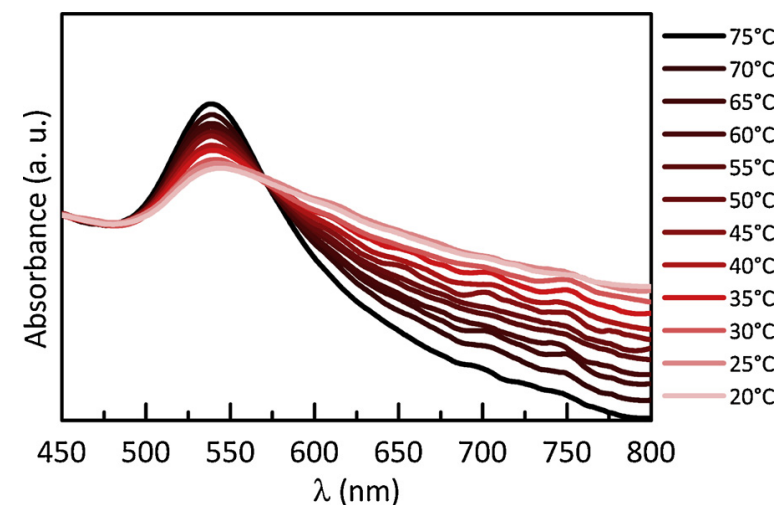

Fig. 4. UV-vis absorption spectra acquired as a function of temperature on the hybrid system composed of the two AuNP dispersions (functionalized with chain-A and chain-B) and the bridge molecules. As the temperature decreases, markers of the NP aggregation, as redshift and increasing peak width, become evident.

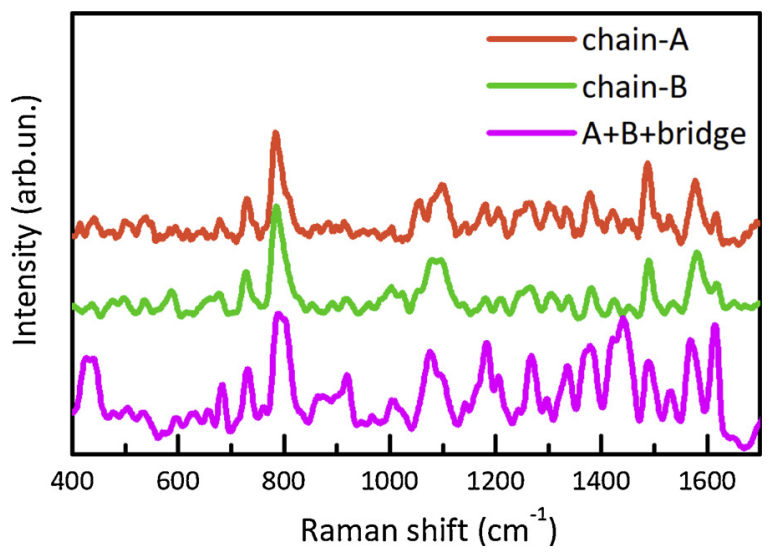

Fig. 5. SERS spectra of the single-chains, chain-A (orange) and chain-B (green), are compared with the SERS spectrum of the combined system $\mathrm{A}+\mathrm{B}+$ bridge (magenta). (For interpretation of the references to color in this figure legend, the reader is referred to the web version of this article.)

bridge-conjugated aggregates. Spectra are normalized to the most intense SERS band, $\sim 784 \mathrm{~cm}^{-1}$.

Despite the spectra in Fig. 5 look quite different, the first information that is derived from the data is that no undoubtedly new peaks originating from the presence of the bridge molecule or the hybridization are observed. This is not surprising since, in principle, spurious base-pairing can occur accidentally also between non-complementary sequences as a consequence of the drying process. This partially prevents the spectroscopic discrimination between the specific hybridizations in the paired system and non-specific bindings, which can be observed also in the single-chain systems [34].

Nevertheless, a remarkable spectral weight redistribution between the various components of the SERS signature can be observed in the spectrum of the bridge-conjugated system, which appears quite different from those of chain-A and chain-B functionalized NPs. These spectral modifications can be related to a partially different orientation of the chains with respect to the NP surface in the case of the conjugated system. Indeed, in the presence of the bridge molecule, we expect the DNA strands to stand upright onto the NP surface, because of the occurred interchain pairing. In the single-chain functionalized NPs, on the other hand, the molecules on the NP surface can move in a relatively more flexible manner, thus their orientation is not strictly determined. The orientation of the DNA molecules is known to critically affect the SERS spectral shape, mostly in the $1200-1600 \mathrm{~cm}^{-1}$ region (high-frequency side of Fig. 5) and in correspondence of the adenine band at $730 \mathrm{~cm}^{-1}$ [22]. Fortunately, the use of thiolated molecules reduces this variability [34], resulting in a less pronounced spectral modification in the present case compared to Refs. [22,33].

As discussed above, a certain variability is expected to affect the high-frequency side of the spectra in Fig. 5 as a consequence of molecular orientation. On the other hand, a close inspection of the SERS band used for spectral normalization, around $784 \mathrm{~cm}^{-1}$, reveals a modified shape for the bridge-conjugated system, compared to the single-chain spectra. It is generally accepted that this band originates from two distinct contributions, one ascribed to the cytosine mode, the other to the phosphate group $[15,20,21,48]$. In particular, the inspection of the results in Refs. $[15,48]$ supports ascribing the low frequency component of the band to the cytosine vibration, and the higher component to the O-P-O stretching mode of the phosphate backbone. To quantify the spectral modification in the three spectra of Fig. 5, we carried out a careful data fitting, deconvolving the contributions to the band into two gaussian functions, centered respectively at $783 \pm 1 \mathrm{~cm}^{-1}$ and $800 \pm 1 \mathrm{~cm}^{-1}$. The ratio between the intensity of the low frequency (cytosine) and high frequency (O-P-O) components nicely correlates with the amount of cytosine in the probed strands 


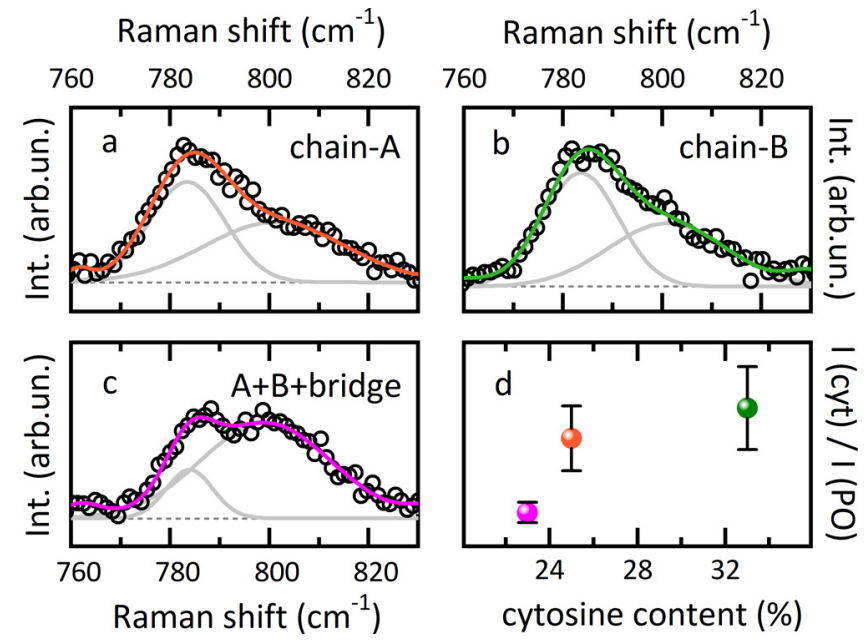

Fig. 6. Gaussian deconvolution of the SERS spectra of chain-A (a), chain-B (b) and bridge-hybridized sample (c) in the $760-830 \mathrm{~cm}^{-1}$ spectral region, where the band assigned to the cytosine and O-P-O vibrations is visible. In panel d, the ratio between the intensity of the low to high frequency components of the band is correlated with the concentration of cytosine in the probed sequences.

(Fig. 6d), thus demonstrating that, despite the discussed sources of variability in the SERS spectral shape from sample to sample, the obtained SERS spectra reflect the DNA composition. The peak discussed in Fig. 6 is far from the typical SERS fingerprint spectral region. Envisioning the use of this system as a biosensor, this will simultaneously enable to detect analytes and to monitor the state and composition of the molecular scaffold that holds the plasmonic nanosystem together.

An aspect to be addressed is the less favorable signal-to-noise ratio that characterizes the spectrum of the $\mathrm{A}+\mathrm{B}+$ bridge conjugated system compared to the spectra of the single-chain samples. This is clearly visible in Figure S6 of the Supplementary Material, where the raw data collected on the single-chain and bridge-conjugated systems are presented along with the smoothed data of Fig. 5. This is consistent with the different orientation of the DNA molecules in the two samples: for single-stranded functionalized NPs, the higher flexibility of DNA with respect to the double-stranded case can result in a more compact arrangement of the molecules in the proximity of the gold surface, where the enhancement is stronger, yielding to a clearer signal. Moreover, since most of the SERS signal arises from interparticle hot-spots, differences can be found in accidental and controlled NP aggregations (i.e. single and double strands).

Support to the hypothesis of a controlled molecular arrangement, orthogonal to the NP surface, in the bridge-mediated aggregates is given by the observation that a more pronounced reproducibility in the spectral intensity is retrieved on the bridge-conjugated system. As a representative example, we show in Figure S7 the most and least intense spectra acquired on a micrometric NP aggregate on the singlechain and bridge-combined samples. Furthermore, we demonstrate the remarkable reproducibility of the spectra of the hybridized system by monitoring the intensity variability among several bridge-mediated aggregates (see Figure S8, Section G in the Supplementary Material). To investigate in detail the relation between the SERS response and the NP aggregation, we carried out high resolution FESEM imaging on both the single-chain AuNP depositions and on the bridge-conjugated aggregates.

Representative results of FESEM microscopy are shown in Fig. 7, where the images acquired on chain-A (panel a) and the $\mathrm{A}+\mathrm{B}+$ bridge (panel b) samples are compared. The morphology of the SERS substrates appears clearly different in the two cases. In the single-stranded case, AuNPs are sparsely and randomly distributed onto the silicon substrate and only a few small aggregates (dimers or trimers) can be found. Conversely, for the bridge-mediated system AuNPs appear organized in large clusters. The zoomed images next to each frame show that the interparticle distance in the chain-A trimer is smaller than the hot-spot size within the A + B + bridge clusters. Remarkably, in the bridge-conjugated substrate, it is possible to recognize a junction between packed AuNPs that can be attributed to the bridge-connected double-stranded DNA chains.

For quantitatively clarifying the role of the nanoarchitecture in the originated SERS intensity and spectral quality, we analyzed the FESEM images in terms of radial autocorrelation function to extrapolate the average interparticle distance, as described in Section I of the Supplementary Material. The analyzed data, reported in Table 1, reveals an average interparticle distance that is almost doubled in the bridge-conjugated aggregates with respect to single-chain functionalized AuNPs. This depends on the semirigid, double helix DNA scaffold holding together the NPs when their aggregation is mediated by the bridge molecule [49]. On the other hand, when the single-chain functionalized NP dispersion is deposited on a glass slide, the aggregation occurs by the uncontrolled self-assembly of the NPs, leading to smaller interparticle distances and more efficient, yet less reproducible hotspots. Consistently, the lower signal revealed in the bridge-conjugated SERS system was accompanied by a higher reproducibility in the overall spectral intensity.

As a final step, we carried out a proof-of-principle sensing test. By employing the bridge-aggregated system as a SERS substrate, we performed the detection of the standard SERS analyte 4-aminothiophenol (4ATP). Details and results of the experiment are discussed in Section $\mathrm{H}$ of the Supplementary Material. A fixed volume of 4ATP solution at a variable concentration was dropcasted on the bridge-aggregated SERS system, dried on a solid substrate. SERS spectra were then measured: by monitoring the intensity as a function of the concentration, a limit of detection lower than $100 \mathrm{nM}$ was retrieved. The enhancement factor was also determined as $(2.2 \pm 0.2) \cdot 10^{5}$. The result is remarkable, considering that the interparticle distance is here quite large $(5.2 \pm 0.6 \mathrm{~nm})$ compared to the usual gap size in SERS hot-spots (see e.g. ref. [45]). In the present case, the molecules are deposited on top of the assembled aggregates to assess the sensing capability of the system as depicted in Fig. 7b. Possible strategies for further improving the enhancement factor could be the choice of shorter DNA sequences, for a more compact assembly. Beside employing the present system as a SERS substrate, we propose the possibility to induce the (reversible) DNA mediated aggregation in presence of analytes by monitoring the temperature of the NP solution. Of course, the presence of additional analytes would result in a modified chemical environment for the DNA hybridization and would result in an additional study.

\section{Conclusions}

In conclusion, we proposed a stable, biocompatible SERS active system based on gold NPs covalently functionalized with specific DNA strands. The advantage of covalent DNA anchoring on the NP surface is twofold: on one hand, we demonstrated that this allows obtaining SERS spectra with reproducible and controlled spectral shapes, proving that the covalent functionalization prevents the accidental physisorption of different, random molecules on the NP surface [18]. On the other hand, the covalent functionalization grants a well-defined orientation of the conjugated DNA strands on the NP surface, thus reducing variabilities in the SERS spectral shape [34] and sterically favoring the DNA hybridization. After functionalizing $60 \mathrm{~nm}$ gold NPs in a buffer solution with two purposely-selected 12-base single-stranded DNA sequences, we induced their aggregation into mesoscopic aggregates by adding to the NP dispersion a complementary, 24-base bridge sequence. We monitored the aggregation of the system as a function of temperature by UV-vis absorption spectroscopy, proving the specificity of the DNA mediated interparticle pairing.

The main features revealed by the DNA-SERS spectra cover a spectral window that is not superimposed to the usual fingerprint 


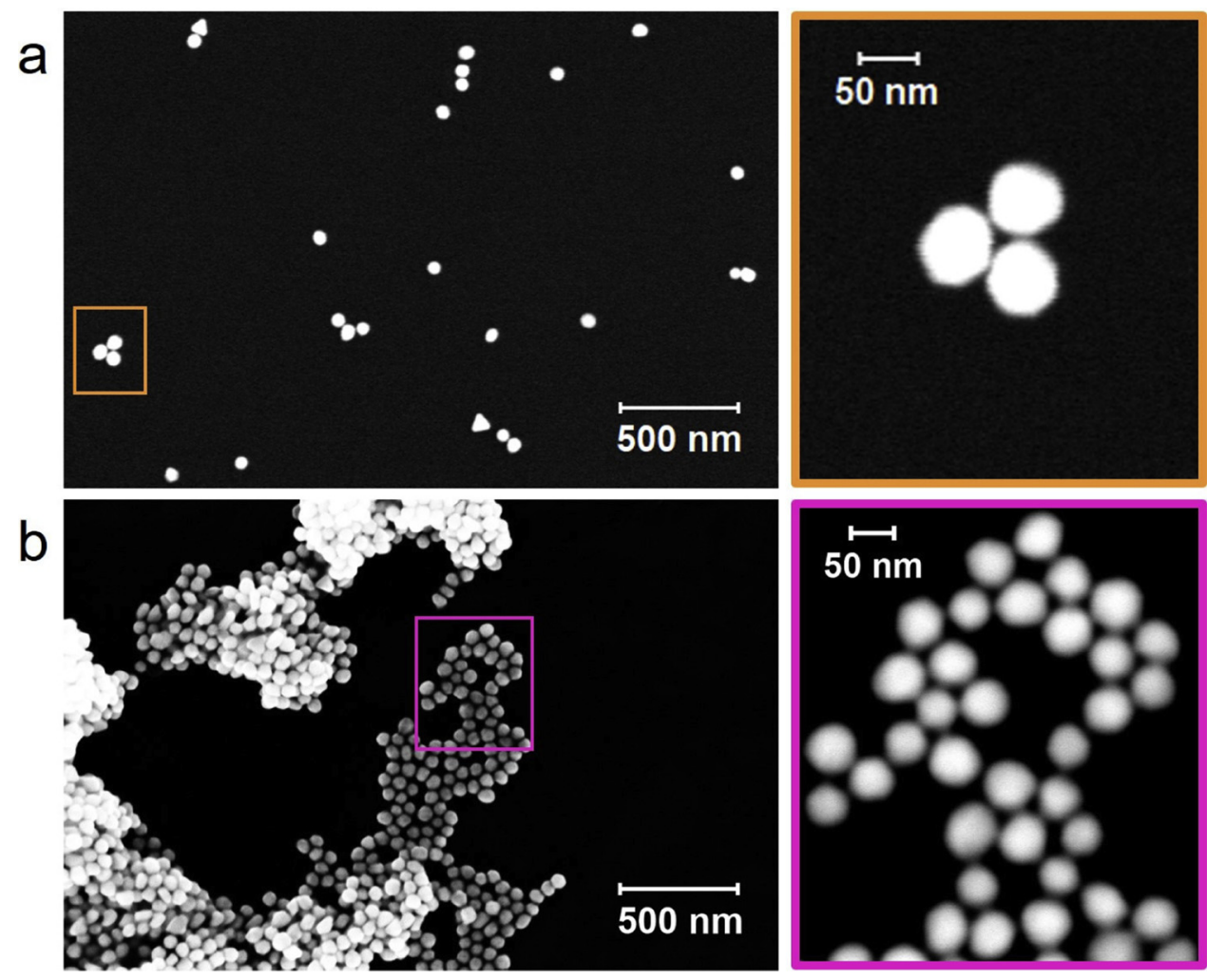

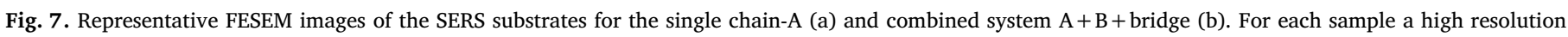
zoom of the highlighted detail is also shown.

Table 1

Inferred hot-spot size within aggregates.

\begin{tabular}{ll}
\hline Sample & Interparticle distance $(\mathrm{nm})$ \\
\hline chain-A & $2.6 \pm 1.1$ \\
chain-B & $3.0 \pm 0.6$ \\
A + B + bridge & $5.2 \pm 0.6$ \\
\hline
\end{tabular}

region of most of the analytes revealed in SERS biosensors [19,39,50]. This, along with the overall low Raman/SERS cross-section of DNA molecules compared to other analytes, particularly those containing aromatic moieties, grants a good contrast for the potential spectroscopic detection of analytes in such a DNA-SERS biosensor. In the present study, the focus was not explicitly on the application of the system as a spectroscopic sensor: instead, we paid more attention to the thorough characterization of the nanosystems from both the composition and the structural point of view. In this respect, all the characteristics of the SERS spectra obtained from the DNA-conjugated nanosystems were justified and interpreted in terms of the system nanoscale architecture.

The spectral shapes in all the systems are very well-defined, as confirmed by repeated measurements, whereas the same consideration does not hold for the SERS intensity. We revealed a higher but less reproducible intensity in the single-chain functionalized systems, while the bridge-conjugated aggregated system features a more pronounced reproducibility. This is ascribed to the NP aggregation process, which is spontaneous and uncontrolled in the single-chain cases, leading to rare and more efficient hot-spots, while it is well predictable and reproducible when the NP aggregation is driven by the specific pairing induced by the bridge molecule. The lower signal-to-noise ratio in the spectra of the bridge-conjugated system is due to the NPs being kept $5.2 \mathrm{~nm}$ far apart by the bridge linking. The results suggest that selecting shorter sequences for such a system can increase the intensity of the DNA-SERS spectra. The spectral shapes have been interpreted in terms of both molecular orientation and DNA strand base composition. We stress that bridge binding results in a reproducible redistribution of the spectral weight in the SERS spectra because of the molecular reorientation. For this reason, despite no specific peak ascribed to the bridge sequence can be identified in the spectrum, the bridge molecule can be detected from the spectral effect of the hybridization. Targeted gene detection is therefore possible by properly selecting the A and $\mathrm{B}$ sequences.

We envision the application of the present system as a stable, versatile biosensor. The advantages of such a system can be numerous. The reproducible SERS enhancement demonstrated from the signal of DNA can be exploited for sensing other molecules, as demonstrated by sensing the 4ATP standard analyte. Here, we have employed the system as a SERS substrate, by dropcasting the analyte on the $\mathrm{A}+\mathrm{B}+$ bridge aggregates. A further possibility that can be considered is inducing the NP aggregation in presence of analytes, to trap them efficiently in the hotspots of the structure. Furthermore, by selecting the proper DNA strands, one can tune the interparticle distance, not only controlling the SERS enhancement but also favoring the trapping and sensing of smaller or bigger molecules. Finally, the base-pairing specificity can be exploited for specific, targeted gene detection.

\section{Authors' contributions}

D. Caprara: Investigation, Formal analysis, Writing - Original Draft.

F. Ripanti: Validation, Supervision, Writing - Review \& Editing.

A. Capocefalo: Investigation, Methodology, Writing - Review \& Editing.

A. Sarra: Investigation

F. Brasili: Investigation, Formal analysis, Writing - Review \& Editing

C. Petrillo: Supervision, Writing - Review \& Editing

C. Fasolato: Conceptualization, Supervision, Writing - Original Draft 


\author{
P. Postorino: Conceptualization, Project administration, Writing - \\ Review \& Editing
}

\section{Funding sources}

The authors acknowledge the Physics Department of Sapienza University for providing access to the CNIS facilities for FESEM measurements. D.C. acknowledges Sapienza University for funding "Avvio alla Ricerca” project 2017.

\section{Conflicts of interest}

The authors have no competing interests to declare.

\section{Acknowledgements}

The authors would like to thank Dr. Francesco Mura for the technical support in FESEM imaging.

\section{Appendix A. Supplementary data}

Supplementary data associated with this article can be found, in the online version, at https://doi.org/10.1016/j.colsurfa.2019.124399.

\section{References}

[1] I. Hamley, Nanostructure fabrication using block copolymers, Nanotechnology 14 (10) (2003) R39.

[2] M. Brust, C.J. Kiely, Some recent advances in nanostructure preparation from gold and silver particles: a short topical review, Colloids Surf. A: Physicochem. Eng. Asp. 202 (2-3) (2002) 175-186.

[3] N.C. Seeman, A.M. Belcher, Emulating biology: building nanostructures from the bottom up, Proc. Natl Acad. Sci. USA 99 (Suppl. 2) (2002) 6451-6455.

[4] R. Luttge, Massively parallel fabrication of repetitive nanostructures: nanolithography for nanoarrays, J. Phys. D: Appl. Phys. 42 (12) (2009) 123001.

[5] F. Watt, A. Bettiol, J. Van Kan, E. Teo, M. Breese, Ion beam lithography and nanofabrication: a review, Int. J. Nanosci. 4 (3) (2005) 269-286.

[6] N.C. Seeman, DNA in a material world, Nature 421 (6921) (2003) 427.

[7] S. Biffi, R. Cerbino, F. Bomboi, E.M. Paraboschi, R. Asselta, F. Sciortino, T. Bellini, Phase behavior and critical activated dynamics of limited-valence DNA nanostars, Proc. Natl. Acad. Sci. USA 110 (39) (2013) 15633-15637.

[8] F. Bomboi, F. Romano, M. Leo, J. Fernandez-Castanon, R. Cerbino, T. Bellini, F. Bordi, P. Filetici, F. Sciortino, Re-entrant DNA gels, Nat. Commun. 7 (2016) 13191.

[9] N. Seeman, H. Sleiman, DNA nanotechnology, Nat. Rev. Mater. 3 (2017), https:// doi.org/10.1038/natrevmats.2017.68.

[10] F. Bomboi, D. Caprara, J. Fernandez-Castanon, F. Sciortino, Cold-swappable DNA gels, Nanoscale 11 (19) (2019) 9691-9697.

[11] P. Kuehler, E.-M. Roller, R. Schreiber, T. Liedl, T. Lohmueller, J. Feldmann, Plasmonic DNA-origami nanoantennas for surface-enhanced Raman spectroscopy, Nano Lett. 14 (5) (2014) 2914-2919.

[12] L. He, M.D. Musick, S.R. Nicewarner, F.G. Salinas, S.J. Benkovic, M.J. Natan, C.D. Keating, Colloidal au-enhanced surface plasmon resonance for ultrasensitive detection of DNA hybridization, J. Am. Chem. Soc. 122 (38) (2000) 9071-9077.

[13] K. Nakatani, S. Sando, I. Saito, Scanning of guanine-guanine mismatches in DNA by synthetic ligands using surface plasmon resonance, Nat. Biotechnol. 19 (1) (2001) 51.

[14] K. Kneipp, H. Kneipp, V.B. Kartha, R. Manoharan, G. Deinum, I. Itzkan, R.R. Dasari, M.S. Feld, Detection and identification of a single DNA base molecule using surfaceenhanced Raman scattering (sers), Phys. Rev. E 57 (6) (1998) R6281.

[15] L. Guerrini, Ž. Krpetić, D. van Lierop, R.A. Alvarez-Puebla, D. Graham, Direct surface-enhanced Raman scattering analysis of DNA duplexes, Angew. Chem. 127 (4) (2015) 1160-1164.

[16] E. Garcia-Rico, R.A. Alvarez-Puebla, L. Guerrini, Direct surface-enhanced Raman scattering (sers) spectroscopy of nucleic acids: from fundamental studies to real-life applications, Chem. Soc. Rev. 47 (13) (2018) 4909-4923.

[17] S. Eustis, M.A. El-Sayed, Why gold nanoparticles are more precious than pretty gold: noble metal surface plasmon resonance and its enhancement of the radiative and nonradiative properties of nanocrystals of different shapes, Chem. Soc. Rev. 35 (3) (2006) 209-217.

[18] F. Domenici, C. Fasolato, E. Mazzi, L. De Angelis, F. Brasili, F. Mura, P. Postorino, F. Bordi, Engineering microscale two-dimensional gold nanoparticle cluster arrays for advanced raman sensing: an afm study, Colloids Surf. A: Physicochem. Eng. Asp. 498 (2016) 168-175.

[19] F. Domenici, A.R. Bizzarri, S. Cannistraro, Sers-based nanobiosensing for ultrasensitive detection of the p53 tumor suppressor, Int. J. Nanomed. 6 (2011) 2033.

[20] L.-J. Xu, Z.-C. Lei, J. Li, C. Zong, C.J. Yang, B. Ren, Label-free surface-enhanced Raman spectroscopy detection of DNA with single-base sensitivity, J. Am. Chem. Soc. 137 (15) (2015) 5149-5154.

[21] A. Torres-Nunez, K. Faulds, D. Graham, R. Alvarez-Puebla, L. Guerrini, Silver colloids as plasmonic substrates for direct label-free surface-enhanced Raman scattering analysis of DNA, Analyst 141 (17) (2016) 5170-5180.

[22] E. Papadopoulou, S.E. Bell, DNA reorientation on au nanoparticles: label-free detection of hybridization by surface enhanced Raman spectroscopy, Chem. Commun. 47 (39) (2011) 10966-10968.

[23] S. Dick, S. Bell, Quantitative surface-enhanced Raman spectroscopy of single bases in oligodeoxynucleotides, Faraday Discuss. 205 (2017) 517-536.

[24] S. Picciolini, D. Mehn, C. Morasso, R. Vanna, M. Bedoni, P. Pellacani, G. Marchesini, A. Valsesia, D. Prosperi, C. Tresoldi, et al., Polymer nanopillar-gold arrays as surface-enhanced Raman spectroscopy substrate for the simultaneous detection of multiple genes, ACS Nano 8 (10) (2014) 10496-10506.

[25] L. He, E. Lamont, B. Veeregowda, S. Sreevatsan, C.L. Haynes, F. Diez-Gonzalez, T.P. Labuza, Aptamer-based surface-enhanced raman scattering detection of ricin in liquid foods, Chem. Sci. 2 (8) (2011) 1579-1582.

[26] O. Neumann, D. Zhang, F. Tam, S. Lal, P. Wittung-Stafshede, N.J. Halas, Direct optical detection of aptamer conformational changes induced by target molecules, Anal. Chem. 81 (24) (2009) 10002-10006.

[27] N.H. Kim, S.J. Lee, M. Moskovits, Reversible tuning of sers hot spots with aptamers, Adv. Mater. 23 (36) (2011) 4152-4156.

[28] W. Fang, S. Jia, J. Chao, L. Wang, X. Duan, H. Liu, Q. Li, X. Zuo, L. Wang, L. Wang, et al., Quantizing single-molecule surface-enhanced raman scattering with dna origami metamolecules, Sci. Adv. 5 (9) (2019) eaau4506.

[29] S.E. Bell, N.M. Sirimuthu, Surface-enhanced Raman spectroscopy (sers) for submicromolar detection of DNA/RNA mononucleotides, J. Am. Chem. Soc. 128 (49) (2006) 15580-15581.

[30] G. Sinha, L.E. Depero, I. Alessandri, Recyclable sers substrates based on au-coated zno nanorods, ACS Appl. Mater. Interfaces 3 (7) (2011) 2557-2563.

[31] L. Wang, Z.-F. Gan, D. Guo, H.-L. Xia, F.T. Patrice, M.E. Hafez, D.-W. Li, Electrochemistry-regulated recyclable sers sensor for sensitive and selective detection of tyrosinase activity, Anal. Chem. 91 (10) (2019) 6507-6513.

[32] J.J. Storhoff, R. Elghanian, R.C. Mucic, C.A. Mirkin, R.L. Letsinger, One-pot colorimetric differentiation of polynucleotides with single base imperfections using gold nanoparticle probes, J. Am. Chem. Soc. 120 (9) (1998) 1959-1964, https:// doi.org/10.1021/ja972332i.

[33] A. Barhoumi, D. Zhang, F. Tam, N.J. Halas, Surface-enhanced Raman spectroscopy of DNA, J. Am. Chem. Soc. 130 (16) (2008) 5523-5529.

[34] N.E. Marotta, K.R. Beavers, L.A. Bottomley, Limitations of surface enhanced Raman scattering in sensing DNA hybridization demonstrated by label-free DNA oligos as molecular rulers of distance-dependent enhancement, Anal. Chem. 85 (3) (2013) 1440-1446.

[35] V.V. Thacker, L.O. Herrmann, D.O. Sigle, T. Zhang, T. Liedl, J.J. Baumberg, U.F. Keyser, DNA origami based assembly of gold nanoparticle dimers for surfaceenhanced Raman scattering, Nat. Commun. 5 (2014) 3448.

[36] I. Fratoddi, I. Venditti, C. Cametti, M.V. Russo, How toxic are gold nanoparticles? The state-of-the-art, Nano Res. 8 (6) (2015) 1771-1799.

[37] O. Bar-Ilan, R.M. Albrecht, V.E. Fako, D.Y. Furgeson, Toxicity assessments of multisized gold and silver nanoparticles in zebrafish embryos, Small 5 (16) (2009) 1897-1910.

[38] C. Heck, Y. Kanehira, J. Kneipp, I. Bald, Placement of single proteins within the sers hot spots of self-assembled silver nanolenses, Angew. Chem. Int. Ed. 57 (25) (2018) 7444-7447.

[39] A. Capocefalo, D. Mammucari, F. Brasili, C. Fasolato, F. Bordi, P. Postorino, F. Domenici, Exploring the potentiality of a sers-active ph nano-biosensor, Front. Chem. 7 (2019) 413.

[40] J. Storhoff, A. Lazarides, R. Mucic, C. Mirkin, R. Letsinger, G. Schatz, What controls the optical properties of DNA-linked gold nanoparticle assemblies? J. Am. Chem. Soc. 122 (19) (2000) 4640-4650, https://doi.org/10.1021/ja993825l.

[41] H.D. Hill, C.A. Mirkin, The bio-barcode assay for the detection of protein and nucleic acid targets using dtt-induced ligand exchange, Nat. Protoc. 1 (2006) 324-336.

[42] H.D. Hill, J.E. Millstone, M.J. Banholzer, C.A. Mirkin, The role radius of curvature plays in thiolated oligonucleotide loading on gold nanoparticles, ACS Nano 3 (2) (2009) 418-424.

[43] J.N. Zadeh, C.D. Steenberg, J.S. Bois, B.R. Wolfe, M.B. Pierce, A.R. Khan, R.M. Dirks, N.A. Pierce, Nupack: analysis and design of nucleic acid systems, J. Comput. Chem. 32 (1) (2011) 170-173.

[44] D. Nečas, P. Klapetek, Gwyddion: an open-source software for spm data analysis, Open Phys. 10 (1) (2012) 181-188.

[45] C. Fasolato, F. Domenici, S. Sennato, F. Mura, L. De Angelis, F. Luongo, F. Costantini, F. Bordi, P. Postorino, Dimensional scale effects on surface enhanced Raman scattering efficiency of self-assembled silver nanoparticle clusters, Appl. Phys. Lett. 105 (7) (2014) 73105.

[46] Y. Fang, N.-H. Seong, D.D. Dlott, Measurement of the distribution of site enhancements in surface-enhanced Raman scattering, Science 321 (5887) (2008) 388-392.

[47] C. Fasolato, S. Giantulli, A. Capocefalo, Y. Toumia, D. Notariello, F. Mazzarda, I. Silvestri, P. Postorino, F. Domenici, Antifolate sers-active nanovectors: quantitative drug nanostructuring and selective cell targeting for effective theranostics, Nanoscale 11 (32) (2019) 15224-15233.

[48] G.J. Thomas Jr., J.M. Benevides, S.A. Overman, T. Ueda, K. Ushizawa, M. Saitoh, M. Tsuboi, Polarized Raman spectra of oriented fibers of a DNA and b DNA: anisotropic and isotropic local Raman tensors of base and backbone vibrations, Biophys. J. 68 (3) (1995) 1073-1088.

[49] A. Japaridze, D. Vobornik, E. Lipiec, A. Cerreta, J. Szczerbinski, R. Zenobi, G. Dietler, Toward an effective control of DNA's submolecular conformation on a surface, Macromolecules 49 (2) (2016) 643-652.

[50] J.Y. Xu, J. Wang, L.T. Kong, G.C. Zheng, Z. Guo, J.H. Liu, Sers detection of explosive agent by macrocyclic compound functionalized triangular gold nanoprisms, J. Raman Spectrosc. 42 (9) (2011) 1728-1735. 